\title{
GLOBAL SMOOTH SOLUTIONS FOR A CLASS OF PARABOLIC INTEGRODIFFERENTIAL EQUATIONS
}

\author{
HANS ENGLER
}

\begin{abstract}
The existence and uniqueness of smooth global large data solutions of a class of quasilinear partial integrodifferential equations in one space and one time dimension are proved, if the integral kernel behaves like $t^{-\alpha}$ near $t=0$ with $\alpha>2 / 3$. An existence and regularity theorem for linear equations with variable coefficients that are related to this type is also proved in arbitrary space dimensions and with no restrictions for $\alpha$.
\end{abstract}

\section{INTRODUCTION}

The goal of this paper is a study of quasilinear partial integrodifferential equations of the following type:

$$
u_{t t}(x, t)-\varphi\left(u_{x}(x, t)\right)_{x}-\int_{0}^{t} a(t-s) \psi\left(u_{x}(x, s)\right)_{x s} d s=f(x, t)
$$

for $0<x<1,0<t \leq T$. We prescribe zero boundary data

$$
u(0, t)=u(1, t)=0 \quad(0 \leq t \leq T)
$$

and initial data

$$
u(x, 0)=u_{0}(x), u_{t}(x, 0)=u_{1}(x) \quad(0 \leq x \leq 1) .
$$

Here $\varphi, \psi: \mathbf{R} \rightarrow \mathbf{R}$ are sufficiently smooth functions, $a:[0, T] \rightarrow \mathbf{R}$ is an integrable function that behaves like $t^{-\alpha}$ near $t=0$ for some $\alpha \in(0,1)$, and $u_{0}, u_{1}, f$ are given functions. Subscripts denote partial derivatives. We are interested in solutions $u$ for which $u_{t t}$ and $u_{x x t}$ are integrable to some sufficiently high power, for data of arbitrary size and for large times. For such solutions, $u_{x t}$ is still continuous, natural uniqueness results hold, and higher smoothness properties can be shown with the aid of a theory of corresponding linear equations. More general linear and quasilinear equations in more than one space dimension will also be studied.

The main results in this paper then imply

a) the existence of such solutions if $\psi^{\prime}$ is positive and bounded, $\varphi$ is Lipschitz continuous, and $a$ is more singular than $t^{-\frac{2}{3}}$ near $t=0$; see Theorem 1.1,

Received by the editors September 22, 1994 and, in revised form, January 13, 1995.

1991 Mathematics Subject Classification. Primary 45K05.

Key words and phrases. Integrodifferential equation, quasilinear, regular solution, global existence, regularity, singular kernel.

Supported by the National Science Foundation under grant \# DMS-9003543 
b) the uniqueness of such solutions if $a$ behaves like $t^{-\alpha}$ for some $\alpha \in(0,1)$, also for related problems in more than one space dimension; see Theorem 5.1,

c) the existence, uniqueness, and continuous dependence on their data of solutions in this class for related linear equations with continuous coefficients, again also in higher space dimensions; see Theorem 2.2 and its corollaries and extensions in section 2 .

Equation (1.1a) is of interest since it is a mathematical idealization of a model for certain problems in nonlinear viscoelasticity. Namely, suppose that $u$ describes the longitudinal displacement of a viscoelastic bar of unit density that occupies the unit interval $[0,1]$ in its stress-free rest position and whose ends are kept fixed. The motion of the bar then is governed by the equation

$$
u_{t t}(x, t)=\sigma_{x}(x, t)+f(x, t)
$$

where $\sigma$ is the one-dimensional stress and $f$ describes body forces. In nonlinear elasticity it is assumed that

$$
\sigma(x, t)=\varphi\left(u_{x}(x, t)\right)
$$

while a simple viscoelastic model of rate type uses the assumption

$$
\sigma(x, t)=\varphi\left(u_{x}(x, t)\right)+\psi\left(u_{x}(x, t)\right)_{t} .
$$

If a constitutive law of the form

$$
\sigma(x, t)=\varphi\left(u_{x}(x, t)\right)+\int_{-\infty}^{t} a(t-s) \psi\left(u_{x}(x, s)\right)_{s} d s
$$

is assumed and if the bar was in the same state of deformation for all $t \in(-\infty, 0]$, then (1.2) and (1.5) lead to (1.1a). Combining (1.2) and (1.3) leads to a nonlinear wave equation for which smooth data are known to develop singularities (discontinuities in $u_{x}$ ) in finite time; see [23]. Combining (1.2) and (1.4) on the other hand results in an equation which is equivalent to a degenerate parabolic system and for which global solutions with the same smoothness as the data can be found, for arbitrary data $([27])$. The viscous damping mechanism that is present in (1.5) is weaker than the one in (1.4), and thus the question arises whether it will prevent the formation of singularities in the solution.

There has been a substantial amount of work on problems of the form (1.1a) or of its integrated version

$$
u_{t}(x, t)-\int_{0}^{t} \varphi\left(u_{x}(x, s)\right)_{x} d s-\int_{0}^{t} a(t-s) \psi\left(u_{x}(x, s)\right)_{x} d s=F(x, t)
$$

with

$$
F(x, t)=u_{1}(x)+\int_{0}^{t} f(x, s) d s-\int_{0}^{t} a(s) \psi\left(u_{0, x}(x)\right)_{x} d s .
$$

We only refer to some of the main results here. If the kernel $a$ is suitably decreasing and smooth up to $t=0$, then local (in time) smooth solutions of $(1.1 \mathrm{a}, \mathrm{b}, \mathrm{c})$ can be 
found just as in the corresponding hyperbolic case with $a=0$. For small initial data, these solutions exist for all times and remain small ([4]), while for large data singularities as in the case with $a=0$ may form in finite time ([5], [13], [19]). Global existence results for smooth small solutions or more generally for small perturbations of arbitrary stationary solutions have been given in the case $a(0)<\infty=-a^{\prime}(0)$ in [21] and [26] and in the case $a(0)=\infty$ in [22], [29] and [32]. Both "singular" cases are of physical interest. For large data and the case $a(0)=\infty$, global weak solutions (with $u_{x}^{2}$ integrable over space and time) are constructed in [20] for the case where $\varphi=0$ and $0<c_{1} \leq \psi^{\prime} \leq c_{2}<\infty$.

More regular solutions (with $u_{x x}$ still square integrable over space and time) are found in [15] and [17] under two different sets of assumptions, namely when $a$ behaves like $t^{-\alpha}$ with $\alpha \geq \frac{1}{2}([17])$ and when the Laplace transform $\hat{a}$ maps the right half plane into a sector in the half plane, under a restriction for $\frac{c_{1}}{c_{2}}$ that depends on the angle of aperture of this sector ([15]). In the latter case, such solutions are also unique ([16]). It turns out that the a priori estimates for the solutions from [15] and [17] are not strong enough to yield continuity of their first space derivatives, a property that turns out to be essential for the treatment in the present paper, since it allows one to write the quasilinear equation for the solution as a linear equation with continuous variable coefficients and to apply a suitable linear regularity theory. The results in [15] and [17] suggest on the other hand that the restriction $\alpha>\frac{2}{3}$ might only be technical and that a result like Theorem 1.1 holds for the entire range $0<\alpha<1$. The existence result in [17] has recently been extended to problems in several space dimensions ([10]). Of course, in higher space dimensions, the gap between solutions with square integrable second space derivatives and with continuous first space derivatives becomes even larger.

For the case where $a(0)<\infty=-a^{\prime}(0)$, global weak solutions were found under the restriction $\varphi=0$ in [25] and for a more general problem that includes (1.1a) in [9]. Some of these papers treat more general and physically more realistic equations, such as the one that results from combining (1.2) with the constitutive law

$$
\sigma(x, t)=\varphi\left(u_{x}(x, t)\right)+\int_{-\infty}^{t} b(t-s) \Psi\left(u_{x}(x, t), u_{x}(x, s)\right) d s
$$

which is equivalent to (1.5) in the special case

$$
b(s)=-a^{\prime}(s), \quad \Psi(U, V)=\psi(U)-\psi(V) .
$$

A detailed overview of the theory until about 1987 can be found in the monograph [31]. The linear theory that underlies these problems is worked out in the monograph [30].

To formulate the existence result for $(1.1 \mathrm{a}, \mathrm{b}, \mathrm{c})$, some notation has to be introduced and some basic assumptions have to be fixed. The usual notation for Sobolev spaces of vector-valued functions will be employed, including the notation for fractional order (Lipschitz -) Sobolev space $W^{s, p}, s>0$ ([1], [33]). The norm on $L^{q}(Q, \mathbf{R})$ or $L^{q}\left(Q, \mathbf{R}^{k}\right)$ will be denoted by $\|\cdot\|_{q}$, if $Q \subset \mathbf{R}^{N}, 1 \leq q \leq \infty$. We shall also write $H^{s}(\ldots)$ for the identical space $W^{s, 2}(\ldots)$. The set $\Omega \subset \mathbf{R}^{n}$ is always assumed to be open, bounded, connected, with $C^{2}$ - smooth boundary $\partial \Omega$ that is locally on one side of $\Omega$. If $n=1$, we shall assume that $\Omega=[0,1]$ and write $I$ for this set. 
Let $1<p, q<\infty$ be numbers satisfying

$$
\frac{2}{p}+\frac{n}{q}<1
$$

Let $T>0$ be given and fixed. For $0<t \leq T$, we define the spaces

$$
\begin{aligned}
Y_{0, q}(\Omega) & =W^{2, q}(\Omega, \mathbf{R}) \cap W_{0}^{1, q}(\Omega, \mathbf{R}), \\
X_{p, q}(t, \Omega) & =L^{p}\left(0, t ; L^{q}(\Omega, \mathbf{R})\right), \\
\tilde{Y}_{p, q}(t, \Omega) & =W^{1, p}\left([0, t], L^{q}(\Omega, \mathbf{R})\right) \cap L^{p}\left(0, t ; Y_{0, q}(\Omega)\right) .
\end{aligned}
$$

Whenever no confusion can arise, we shall write $Y_{0}$ for $Y_{0, q}(\Omega), X(t)$ for $X_{p, q}(t, \Omega)$, and $\tilde{Y}(t)$ for $\tilde{Y}_{p, q}(t, \Omega)$, with norms $\|\cdot\|_{X(t)}$ etc. Similarly, we shall write $\|\cdot\|_{L^{q}(t)}$ etc. If $t=T$, we shall simply write $X, \tilde{Y}$.

Next, let $0<\alpha<1$ be fixed, let

$$
a_{\alpha}(t)=t^{-\alpha} \quad(0<t \leq T)
$$

and define

$$
K(\alpha)=\left\{a:(0, T] \rightarrow \mathbf{R} \mid a=a_{\alpha}+c * a_{\alpha} \quad \text { with } \quad c \in L^{1}(0, T ; \mathbf{R})\right\},
$$

where $v * w(t)=\int_{0}^{t} v(t-s) w(s) d s$ denotes the usual convolution of two functions that are supported on the positive half line.

Our main result on $(1.1 \mathrm{a}, \mathrm{b}, \mathrm{c})$ then is the following.

Theorem 1.1. Let $a \in K(\alpha)$ with $\frac{2}{3}<\alpha<1$, satisfying for some $M>0$

$$
a(t)>0, a^{\prime}(t) \leq 0, a^{\prime \prime}(t) \geq M a^{\prime}(t) \quad(0<t \leq T)
$$

in the sense of distributions and for some $c_{0}>0$ and $\delta_{0}>0$

$$
a^{\prime}(t) \leq-c_{0} t^{-1-\alpha} \quad\left(0<t \leq \delta_{0}\right) .
$$

Let $\varphi, \psi \in C^{2}(\mathbf{R}, \mathbf{R})$ such that for some $L>0$ and all $r \in \mathbf{R}$

$$
\begin{gathered}
0<\psi^{\prime}(r) \leq L,\left|\varphi^{\prime}(r)\right| \leq L \psi^{\prime}(r), \int_{0}^{r} \varphi(\rho) d \rho \geq-L, \\
|\psi(r)| \rightarrow \infty \quad \text { as }|r| \rightarrow \infty .
\end{gathered}
$$

With $p, q$ such that $\frac{2}{p}+\frac{1}{q}<1$, let $u_{0}, u_{1} \in Y_{0, q}(I)$, and let $f: I \times[0, T] \rightarrow \mathbf{R}$ be of the form

$$
f(x, t)=\left\{\begin{array}{l}
a * f_{0}(x, t) \quad \text { if } \quad p(1-\alpha)<1, \\
a * f_{0}(x, t)-\varphi\left(u_{0, x}(x)\right)_{x} \quad \text { if } \quad p(1-\alpha)>1
\end{array}\right.
$$

with $f_{0} \in X_{p, q}(T, I)$. Then there exists a solution $u$ of $(1.1 \mathrm{a}, \mathrm{b}, \mathrm{c})$ with $u_{t} \in$ $\tilde{Y}_{p, q}(T, I)$. This solution is unique in $\tilde{Y}_{p, q}(T, I)$.

The proof of this theorem will be given in section 3 (a priori estimates), section 4 (fixed point argument for existence), and section 5 (uniqueness), after first deriving 
results on linear equations with variable coefficients in section 2. Some comments should serve to explain this result and its context.

a) Solutions of $(1.1 \mathrm{a}, \mathrm{b}, \mathrm{c})$ with better space or time regularity can be found by differentiating (1.1a) with respect to $x$ or $t$ and applying the regularity theory of section 2 to the resulting equations for derivatives of $u$, as long as the data permit this.

b) On the other other hand, it is not clear how to construct global solutions that are only in $\tilde{Y}_{p, q}(T, I)$ for arbitrary data. Such functions would still solve the integrated equation (1.6) with data (1.7) and can be expected to exist for $u_{1} \in L^{q}(I, \mathbf{R})$ and for less regular $f$. With the methods of sections 2 and 5 , a unique local solution can be found, but it is not clear how to obtain the necessary a priori estimates to continue solutions in this weaker class for all times.

c) The sign assumption for $\psi^{\prime}$ is essential, but no lower bound is needed, since $u_{x}$ can be bounded pointwise in terms of $L$ only. No sign assumption for $\varphi^{\prime}$ is needed, since the term with $\varphi$ is really a perturbation term for the time-dependent problem (1.1a). In particular, the equation for the rest state

$$
-\varphi\left(u_{\infty, x}\right)_{x}=f_{\infty}
$$

may have solutions with discontinuous first derivatives. For all finite times, however, the solutions in Theorem 1.1 have continuous first space derivatives.

d) Assumption (1.17) probably reflects a genuine property of the underlying physical problem. To see this, one notes first that if $p(1-\alpha)>1$, then $f$ is actually Hölder continuous with values in $L^{q}(I)$, by well-known properties of Abel integral operators ([12]). In this case, property (1.17) says that the viscoelastic body must be in static equilibrium at $t=0$, and solutions are found with "smooth" behavior near $t=0: u_{t t}$ is as smooth as $f$. The property also stipulates that solutions that are spatially "rough" (small $q$ ) must have good temporal regularity. On the other hand, if for $t=0$ the body forces do not balance the stress in the initial configuration $u_{0}$, then only the existence of solutions with small $p$ is asserted; in this case, $u_{t t} \in L^{r}\left(0, T ; L^{q}(I)\right)$ with $\frac{1}{r}=\frac{1}{p}-1+\alpha$, allowing weak singularities for the acceleration. At the same time, solutions can only be found that have better spatial smoothness ( $q$ large). In any case, the temporal smoothness of the solution is actually better than asserted in the theorem.

e) The restrictive assumption $\frac{2}{3}<\alpha<1$ is probably technical, but it is not clear how to weaken it. What one needs are new a priori estimates that allow one to bound a modulus of continuity of $u_{x}$ for any solution. An interesting method for obtaining $L^{\infty}$ - bounds for $u_{x}$ that was first proposed in [6] for nonlinear wave equations has been extended to integrodifferential equations in [18], allowing the construction of weak solutions via compensated compactness methods. It is intriguing that a slightly stronger estimate (modulus of continuity) would allow the construction of smooth solutions.

f) Uniqueness of solutions holds in a larger class of solutions and without restrictions on the exponent $\alpha$, see Theorem 5.1. The uniqueness proof given there relies on the regularity theory developed in section 2 ; hence it is not entirely satisfactory. For more elementary uniqueness proofs in certain situations that extend to weaker solution classes, see [16] and [17]. 
We finally quote a result on linear equations with constant coefficients from [29] (see also [28]) that will be used in the next section. Let $\Delta$ denote the Laplacian, let $a_{\alpha}(t)=t^{-\alpha}$ as before, and let $\frac{2}{p}+\frac{n}{q}<1$.

Theorem 1.2. Consider the equation

$$
u_{t}(x, t)-\int_{0}^{t} a_{\alpha}(t-s)(\Delta u(x, s)+f(x, s)) d s=0 \quad(x \in \Omega, 0<t \leq T)
$$

with initial data $u(\cdot, 0)=0$ in $\Omega$ and boundary data $u=0$ on $\partial \Omega \times[0, T]$. For any $f \in X_{p, q}(T, \Omega)$ there exists a unique solution $u \in \tilde{Y}_{p, q}(T, \Omega)$, and the estimate holds

$$
\|u\|_{\tilde{Y}} \leq C^{*}\|f\|_{X}
$$

with $C^{*}$ depending only on $\Omega, p, q, \alpha$.

\section{LINEAR PROBLEMS WITH VARIABLE COEFFICIENTS}

Let $a \in K(\alpha)$ be a kernel function as in the previous section, and let $B: \bar{\Omega} \times$ $[0, T] \rightarrow \mathbf{R}_{s y m}^{n, n}$ be a matrix valued function. In this section, we want to study the integrodifferential equation

$$
u_{t}(x, t)-\int_{0}^{t} a(t-s)\left(B(s, x): \nabla^{2} u(x, s)+f(x, s)\right) d s=0
$$

on $\Omega \times[0, T]$ with initial data

$$
u(\cdot, 0)=u_{0}
$$

and boundary data

$$
u(x, t)=0 \quad((x, t) \in \partial \Omega \times[0, T]) .
$$

Here $\nabla^{2} w$ is the Hessian matrix of the scalar function $w$, and $C: D=\operatorname{trace} C D^{T}=$ $\sum_{i, j} c_{i j} d_{i j}$ is the contraction of two matrices with the same dimension. Let $1<$ $p, \quad q<\infty$ with $\frac{2}{p}+\frac{n}{q}<1$, and let the spaces $Y_{0}=Y_{0, q}(\Omega), X=X_{p, q}(T, \Omega), \tilde{Y}=$ $\tilde{Y}_{p, q}(I, \Omega)$ be defined as in the previous section. The goal is to construct unique solutions $u \in \tilde{Y}$ for any given $u_{0} \in Y_{0}$ and $f \in X$ and to prove an estimate of the form

$$
\|u\|_{\tilde{Y}} \leq C_{0}\|f\|_{X}
$$

for the case $u_{0}=0$, with $C_{0}$ not depending on $f$.

Note that $u$ solves $(2.1 \mathrm{a}, \mathrm{b}, \mathrm{c})$ iff $v=u-u_{0}$ solves an equation of the same form with $v(\cdot, 0)=0$ and with $f$ replaced by $\tilde{f}$, where $\tilde{f}(x, t)=f(x, t)+B(x, t): \nabla^{2} u_{0}(x)$. For reasonably regular $B$, it is thus sufficient to solve the problem with zero initial data, as long as the initial data are from the space $Y_{0}$, and in the remainder of this section, $u_{0}=0$ will be assumed, i.e. (2.1b) will be replaced with

$$
u(x, 0)=0 \quad(x \in \Omega) .
$$


Correspondingly, we look for solutions in the space

$$
Y=\{u \in \tilde{Y} \mid u(\cdot, 0)=0\}
$$

with the norm inherited from $\tilde{Y}$. Given a solution theory in $Y$ (such as the one constructed in this paper), one can still solve the general problem $(2.1 \mathrm{a}, \mathrm{b}, \mathrm{c})$ in $\tilde{Y}$ for general initial data $u_{0} \in Y_{0}$, and the estimate $\|u\|_{\tilde{Y}} \leq C\left(\left\|u_{0}\right\|_{Y_{0}}+\|f\|_{X}\right)$ holds. However, one can actually solve the problem in $\tilde{Y}$ for initial data from a trace class that is somewhat larger than $Y_{0}$; see e.g. [7]. Similarly, problems with inhomogeneous boundary data on $\partial \Omega \times[0, T]$ can be solved by subtracting suitable extensions of these data; to determine the optimal data classes for the solutions that are constructed here, trace results must be proved. These possible generalizations will not be pursued in the present paper.

We first show a standard perturbation principle that holds whenever a maximal regularity result is known. It is formulated only for the equation (2.1a), but it obviously also holds for more general equations with several integral terms, lower order terms, etc., such as those discussed below.

Theorem 2.1. Suppose $a$ and $B$ are such that (2.1a,c,d) has a solution $u$ for all $f \in X$ and such that (2.2) holds. Then for any bounded linear operator $L: Y \rightarrow X$ that satisfies

$$
C_{0}\|L\|_{L(Y, X)}<1
$$

the problem

$$
u_{t}(x, t)-\int_{0}^{t} a(t-s)\left(B(s, x): \nabla^{2} u(x, s)+L u(x, s)+f(x, s)\right) d s=0
$$

on $\Omega \times[0, T]$ with initial data (2.1d) also has a unique solution $\tilde{u} \in Y$ for any $f \in X$, and these estimates hold:

$$
\begin{aligned}
\|u-\tilde{u}\|_{Y} & \leq \frac{C_{0}\|L\|_{L(Y, X)}}{1-C_{0}\|L\|_{L(Y, X)}}\|u\|_{Y}, \\
\|\tilde{u}\|_{Y} & \leq \frac{C_{0}}{1-C_{0}\|L\|_{L(Y, X)}}\|f\|_{X} .
\end{aligned}
$$

Proof. Given $L$ and $f$, we seek $\tilde{u}$ as a fixed point of the operator $\mathbf{S}$ that maps $w \in Y$ to $v \in Y$, the unique solution of

$$
v_{t}(x, t)-\int_{0}^{t} a(t-s)\left(B(x, s): \nabla^{2} v(x, s)+L w(x, s)+f(x, s)\right) d s=0
$$

with initial and boundary conditions as in $(2.1 \mathrm{c}, \mathrm{d})$. It follows from $(2.2)$ that

$$
\left\|\mathbf{S} w_{1}-\mathbf{S} w_{2}\right\|_{Y} \leq C_{0}\|L\|_{L(Y, X)}\left\|w_{1}-w_{2}\right\|_{Y} .
$$

The operator $\mathbf{S}$ therefore has a unique fixed point $\tilde{u}$. Subtracting the fixed point equation that must hold for $\tilde{u}$ from the equation for $u$ and applying (2.2), one obtains

$$
\|u-\tilde{u}\|_{Y} \leq C_{0}\|L\|_{L(Y, X)}\|\tilde{u}\|_{Y} \leq C_{0}\|L\|_{L(Y, X)}\left(\|u-\tilde{u}\|_{Y}+\|u\|_{Y}\right)
$$


from which (2.4) follows. Combining (2.4) with (2.2) one obtains (2.5).

This result shows in particular that perturbations of the coefficients of $(2.1 \mathrm{a}, \mathrm{c}, \mathrm{d})$ that are small in the $L^{\infty}$ - norm still result in problems that can be solved in the same sense, and that solutions depend continuously on the perturbed coefficients in this norm. Obviously, the same result for small perturbations is generally true for all problems for which an estimate of the form (2.2) is known to hold. To obtain results for "large" perturbations, however, one needs a separate argument. This is given next, and it is the main result of this section.

Theorem 2.2. Suppose that $B: \bar{\Omega} \times[0, T] \rightarrow \mathbf{R}_{\text {sym }}^{n, n}$ is continuous, with $B(x, t)$ positive definite for all $(x, t)$, and that $a(t)=a_{\alpha}(t)=t^{-\alpha}$ with $0<\alpha<1$. Then for all $f \in X$ there exists a unique function $u \in Y$ that satisfies $(2.1 \mathrm{a}, \mathrm{c}, \mathrm{d})$. The estimate (2.2) holds with a constant $C_{0}$ that depends on $T, \Omega, \alpha$, the infimum of all eigenvalues of $B(x, t)$ as $(x, t)$ varies in $\Omega \times[0, T]$, and the modulus of continuity of $B$.

The proof of Theorem 2.2 will be given below.

We next discuss some extensions to problems with more general kernels, including kernels with weaker singularities, and with lower order terms. Let $N: Y \rightarrow X$ be a given linear operator, with the following additional property:

$(\mathrm{V})$

$\forall \epsilon>0 \exists c_{\epsilon} \in L^{1}(0, T) \forall t \forall u \in Y \quad\|N(u)\|_{X(t)} \leq \epsilon\|u\|_{Y(t)}+\int_{0}^{t} c_{\epsilon}(t-s)\|u\|_{Y(s)} d s$.

The spaces $X(t)=X_{p, q}(t, I)$ etc. have been defined in section 1, and their norms $\|\cdot\|_{X(t)}$ etc. are to be interpreted as seminorms on the spaces $X=X(T)$. Evidently this property implies that the values of $N(u)$ on any cylinder $\Omega \times[0, t]$ only depend on the behavior of $u$ on the same set.

Theorem 2.3. With the assumptions and notation of Theorem 2.2, let $N: Y \rightarrow X$ be a linear operator satisfying $(\mathrm{V})$. Then for any $f \in X$ there exists a unique solution $u \in Y$ of

$$
u_{t}(x, t)-\int_{0}^{t} a(t-s)\left(B(s, x): \nabla^{2} u(x, s)+N(u)(x, s)+f(x, s)\right) d s=0
$$

a.e. in $\Omega \times[0, T]$ with initial data (2.1d). Moreover, with a constant $C_{1}$ that depends on $C_{0}$ and on the family of kernels $c_{\epsilon}$ in $(\mathrm{V})$, the estimate

$$
\|u\|_{Y} \leq C_{1}\|f\|_{Y}
$$

is true for any solution $u$ with data $f$, and $u \in Y$ depends continuously on $N \in$ $L(Y, X)$ in the norm topology.

Proof of Theorem 2.3. For $w \in Y$, we define $u=\mathbf{T} w \in Y$ as the solution of the problem

$$
u_{t}(x, t)-\int_{0}^{t} a(t-s)\left(B(s, x): \nabla^{2} u(x, s)+N(w)(x, s)+f(x, s)\right) d s=0
$$


with initial data (2.1d). We then obtain for any $0 \leq t \leq T$

$$
\begin{aligned}
\|\mathbf{T} v-\mathbf{T} w\|_{Y(t)} & \leq C_{0}\|N(v)-N(w)\|_{X(t)} \\
& \leq \epsilon C_{0}\|v-w\|_{Y(t)}+\int_{0}^{t} C_{0} c_{\epsilon}(t-s)\|v-w\|_{Y(s)} d s
\end{aligned}
$$

with $\epsilon$ still arbitrary. For $M>0$, we define the equivalent norm on $Y$

$$
\|u\|_{M}=\sup _{0 \leq t \leq T} e^{-M t}\|u\|_{Y(t)} .
$$

Then (2.10) implies that for any such $M$

$$
e^{-M t}\|\mathbf{T} v-\mathbf{T} w\|_{Y(t)} \leq \epsilon C_{0}\|v-w\|_{M}+\int_{0}^{t} C_{0} e^{-M(t-s)} c_{\epsilon}(t-s)\|v-w\|_{M} d s
$$

and thus

$$
\|\mathbf{T} v-\mathbf{T} w\|_{M} \leq\left(\epsilon C_{0}+\int_{0}^{T} C_{0} e^{-M s} c_{\epsilon}(s) d s\right)\|v-w\|_{M} .
$$

Choosing first $\epsilon$ sufficiently small and then $M$ sufficiently large implies that $\mathbf{T}$ is a contraction in the norm $\|\cdot\|_{M}$ and thus has a unique fixed point which is the desired solution. The results on the dependence on $f$ and $N$ follow by standard arguments or from Theorem 2.1.

Theorem 2.3 allows to solve equations such as

$$
u_{t}(x, t)-\int_{0}^{t}\left(a_{\alpha}(t-s) B(s, x): \nabla^{2} u(x, s)+h(x, s): \nabla^{2} u(x, s)+f(x, s)\right) d s=0
$$

if merely $h \in L^{\infty}\left(\Omega \times[0, T], \mathbf{R}_{s y m}^{n, n}\right)$, since the integral involving $h$ can be written as

$$
\begin{aligned}
& \int_{0}^{t} h(x, s): \nabla^{2} u(x, s) d s \\
& \quad= \frac{\sin \alpha \pi}{\pi} \int_{0}^{t} a_{\alpha}(t-s) \int_{0}^{s} a_{1-\alpha}(s-\tau) h(x, \tau): \nabla^{2} u(x, \tau) d \tau d s \\
& \quad=\int_{0}^{t} a_{\alpha}(t-s) N(u)(x, s) d s .
\end{aligned}
$$

Clearly, this operator $N$ satisfies (V).

The result also allows more general perturbations of the kernel function $a_{\alpha}$, namely its replacement with general kernels $a=a_{\alpha}+c * a_{\alpha} \in K(\alpha)$, where $c \in$ $L^{1}(0, T ; \mathbf{R})$. In fact, no new difficulty arises if one allows kernels of the form $a=$ $a_{\alpha}+d k * a_{\alpha}$, where $k:[0, T] \rightarrow \mathbf{R}$ has bounded variation and is continuous at 0 , a type of perturbation that is used in [30]. The problem with $a_{\alpha}$ replaced by $a$ in (2.1a) is of the form (2.7) if $N$ is defined as

$$
N(u)(x, t)=\int_{0}^{t} c(t-s) B(x, s): \nabla^{2} u(x, s) d s,
$$

and obviously $N$ satisfies (V). We thus obtain 
Corollary 2.4. The result of Theorem 2.2 remains true if $a \in K(\alpha)$.

In particular, kernels of the form

$$
a(t)=a_{\alpha}(t) d(t)
$$

with $d \in W^{1,1}([0, T], \mathbf{R})$ and $d(0)=1$ are allowed, as Lemma A.1 shows.

In addition to such lower order integral terms, also lower order differential operators are allowed. For example, consider the operator

$$
N(u)(x, t)=\mathbf{b}(x, t)^{T} \nabla u(x, t)+c(x, t) u(x, t),
$$

with $\mathbf{b}: \Omega \times[0, T] \rightarrow \mathbf{R}^{n}$. We assume that $b_{i}, c \in X$ for all $i$ and that $\left\|b_{i}\right\|_{X},\|c\|_{X}$ $\leq M$.

Corollary 2.5. The operator $N$ satisfies $(\mathrm{V})$, with $c_{\epsilon}(t)=C_{\epsilon}$ depending only on $M$.

Proof. Let $C^{1,0}=\left\{u \mid \nabla u \in C\left(\bar{\Omega} \times[0, T], \mathbf{R}^{n}\right)\right\}$. By Lemma A.3, there are continuous imbeddings

$$
Y \subset C^{1,0} \subset X
$$

with the left imbedding being completely continuous. Thus for any $\epsilon$ there exists $C(\epsilon)$ such that for all $u \in Y$

$$
\|u\|_{\infty}+\|\nabla u\|_{\infty} \leq \epsilon\|u\|_{Y}+C(\epsilon)\|u\|_{X}
$$

see e.g. [24]. Now let $0<t \leq T$, and let $\delta>0$ be arbitrary. Then

$$
\begin{aligned}
\|N(u)\|_{X(t)} & \leq\|N(u)\|_{X} \leq M\left(\|u\|_{\infty}+\|\nabla u\|_{\infty}\right) \\
& \leq \delta M\|u\|_{Y}+M C(\delta)\|u\|_{X} .
\end{aligned}
$$

Define $\tilde{u} \in Y$ by

$$
\tilde{u}(\cdot, s)=\left\{\begin{array}{l}
u(\cdot, s) \quad(s \leq t) \\
u(\cdot, 2 t-s) \quad(t<s \leq T)
\end{array}\right.
$$

where $u(\cdot, s)$ is set equal to zero for $s<0$. Then $u$ and $\tilde{u}$ agree on $\Omega \times[0, t]$ and $\|\tilde{u}\|_{X} \leq 2\|u\|_{X(t)},\|\tilde{u}\|_{Y} \leq 2\|u\|_{Y(t)}$. Therefore

$$
\begin{aligned}
\|N(u)\|_{X(t)}=\|N(\tilde{u})\|_{X(t)} & \leq \delta M\|\tilde{u}\|_{Y}+M C(\delta)\|\tilde{u}\|_{X} \\
& \leq 2 \delta M\|u\|_{Y(t)}+2 M C(\delta)\|u\|_{X(t)} \\
& \leq 2 \delta M\|u\|_{Y(t)}+2 M C(\delta) \int_{0}^{t}\|u\|_{Y(s)} d s
\end{aligned}
$$

since $\left\|u_{t}\right\|_{X(t)} \leq\|u\|_{Y(t)}$. Property $(\mathrm{V})$ therefore holds with $c_{\epsilon}(t)=2 M C(\epsilon /(2 M))$.

In particular, the $Y$ - norm of the solution depends only on the $X$ - norm of the coefficients $b_{i}, c$, and the solution depends continuously on the coefficients in these topologies. This property will be used in section 4 .

For the proof of Theorem 2.2, the following observation will be used. 
Lemma 2.6. Let $T_{0}>0,0<c_{0}<c_{1}<\infty$ be fixed. Then Theorem 1.2 also holds if the Laplacian is replaced with an elliptic operator with constant coefficients of the form $A: \nabla^{2}=\sum_{i, j} a_{i j} \frac{\partial^{2}}{\partial x_{i} \partial x_{j}}$, where the matrix $A=\left(a_{i j}\right)_{1 \leq i, j \leq n}$ is symmetric and positive definite. The constant $C$ in (1.20) can be chosen independently of $T$ and of $A$ as long as $0<T \leq T_{0}$ and $A$ has all eigenvalues between $c_{0}$ and $c_{1}$.

Proof. After a rotation and a stretch of the independent coordinates, the elliptic operator becomes the Laplacian, such that the existence assertion follows trivially. Also, estimate (1.20) holds with some constant $C(A, T)$. This constant does not depend on $T$ as long as $T \leq T_{0}$ since the solution of (1.19) on any cylinder $\Omega \times[0, T], T \leq T_{0}$, depends only on $f$ restricted to $\Omega \times[0, T]$. For the second assertion, Theorem 2.1 implies that the best constant $C(A, T)$ in (1.20) is an upper semicontinuous function of the coefficient matrix. Since the set of all symmetric matrices with eigenvalues between $c_{0}$ and $c_{1}$ is compact, the assertion follows.

Lemma 2.7. The assertion of Theorem 2.2 is true if $B$ depends only on $x \in \bar{\Omega}$ and not on $t \in[0, T]$, with the constant $C_{0}$ depending on bounds for the spectra for $B(x)(x \in \bar{\Omega})$, the modulus of continuity of $B, \Omega, \alpha, p, q$, and a bound for $T$.

Proof. For $0 \leq \sigma \leq 1$ and $x \in \bar{\Omega}$, define

$$
B_{i j}^{\sigma}(x)=(1-\sigma) \delta_{i j}+\sigma B_{i j}(x) \quad(1 \leq i, j \leq n)
$$

where $\delta_{i j}$ is the Kronecker symbol. Let $P_{\sigma}$ be the problem of solving $(2.1 \mathrm{a}, \mathrm{c}, \mathrm{d})$ with $B$ replaced by $B^{\sigma}$. Theorems 1.1 and 2.1 then imply the following facts about these problems.

a) $P_{0}$ is uniquely solvable, and estimate (2.2) holds with some constant $C_{0}$.

b) If $P_{\sigma}$ is uniquely solvable for some $\sigma$ and if (2.2) holds with some constant $C_{\sigma}$, then $P_{\sigma^{\prime}}$ is uniquely solvable for all $\sigma^{\prime}$ with $C_{\sigma} K\left|\sigma-\sigma^{\prime}\right|<1$, and (2.2) holds with

$$
C_{\sigma^{\prime}} \leq \frac{C_{\sigma}}{1-C_{\sigma} K\left|\sigma-\sigma^{\prime}\right|}
$$

Here $K$ is some universal constant depending on $n$ and on $\|B\|_{\infty}$.

Put differently, the maximal interval containing 0 of those $\sigma \in[0,1]$ for which $P_{\sigma}$ can be solved with some constant $C_{\sigma}<\infty$ is open in [0,1], and if the interval does not contain $\sigma=1$, then $C_{\sigma}$ must go to infinity at its right endpoint. If we can prove that (2.2) holds with a constant $\bar{C}$ that is independent of $\sigma$ whenever a solution of $P_{\sigma}$ exists, then it follows that all problems $P_{\sigma}$ actually can be solved for $0 \leq \sigma \leq 1$. Taking $\sigma=1$, the assertion of the lemma follows.

To prove this a priori estimate, let $\sigma$ be fixed, and let $v=v^{\sigma}$ be a solution of $P_{\sigma}$. Choose a $C^{2}$ - smooth partition of unity $\left(\phi_{k}\right)_{1 \leq k \leq N(m)}$ for $\bar{\Omega}$, with $0 \leq \phi_{k} \leq 1$ 
and $\operatorname{diam}\left(\operatorname{supp}\left(\phi_{k}\right)\right) \leq m^{-1}$ for all $k$. Set

$$
\begin{aligned}
D_{m} & =\max _{k}\left\|\nabla \phi_{k}\right\|_{\infty}, \\
E_{m} & =\max _{k}\left\|\nabla^{2} \phi_{k}\right\|_{\infty}, \\
B_{i j}^{\sigma, k} & =\left(\int_{\Omega} \phi_{k}(x) d x\right)^{-1} \int_{\Omega} \phi_{k}(x) B_{i j}^{\sigma}(x) d x, \\
v^{\sigma, k}(x, t) & =\phi_{k}(x) v^{\sigma}(x, t), \\
f^{k}(x, t) & =\phi_{k}(x) f(x, t), \\
F^{\sigma, k}(x, t) & =\left(B^{\sigma}(x)-B^{\sigma, k}\right): \nabla^{2} v^{\sigma, k}(x, t), \\
G^{\sigma, k}(x, t) & =B^{\sigma}(x):\left(\nabla^{2} \phi_{k}(x) v^{\sigma}(x, t)+2 \nabla \phi_{k}(x)\left(\nabla v^{\sigma}(x, t)\right)^{T}\right) .
\end{aligned}
$$

Then $v^{\sigma, k}$ is a solution of

$$
\begin{aligned}
v_{t}^{\sigma, k}(x, t)-\int_{0}^{t} a_{\alpha}(t-s)\left(B^{\sigma, k}: \nabla^{2} v^{k}(\right. & x, s)+f^{k}(x, s) \\
& \left.+G^{\sigma, k}(x, s)+F^{\sigma, k}(x, s)\right) d s=0 .
\end{aligned}
$$

By Lemma 2.6, we have an estimate for all $0 \leq t \leq T$ :

$$
\left\|v^{\sigma, k}\right\|_{Y(t)} \leq C^{*}\left(\left\|f^{k}\right\|_{X}+\left\|F^{\sigma, k}\right\|_{X(t)}+\left\|G^{\sigma, k}\right\|_{X(t)}\right)
$$

with $C^{*}$ independent of $k, \sigma, t$, and the $\phi_{k}$. Next,

$$
\left\|F^{\sigma, k}\right\|_{X(t)} \leq \max _{x \in \operatorname{supp}\left(\phi_{k}\right)}\left\|B^{\sigma, k}-B^{\sigma}(x)\right\|\left\|v^{\sigma, k}\right\|_{Y(t)} \leq \omega(m)\left\|v^{\sigma, k}\right\|_{Y(t)}
$$

with some $\omega(m)$ that goes to zero as $m \rightarrow \infty$, depending only on the modulus of continuity of $B$, and

$$
\begin{aligned}
\left\|G^{\sigma, k}\right\|_{X(t)} & \leq 2 K D_{m}\left\|\nabla v^{\sigma}\right\|_{X(t)}+K E_{m}\left\|v^{\sigma}\right\|_{X(t)} \\
& \leq 2 K D_{m}\left(\epsilon\left\|v^{\sigma}\right\|_{Y(t)}+C_{\epsilon}\left\|v^{\sigma}\right\|_{X(t)}\right)+K E_{m}\left\|v^{\sigma}\right\|_{X(t)},
\end{aligned}
$$

where $K=\|B\|_{\infty}, \epsilon>0$ is arbitrary, and $C_{\epsilon}>0$, by applying a calculus inequality ([10]). Combining $(2.21-2.23)$ results in

$$
\begin{aligned}
\left\|v^{\sigma, k}\right\|_{Y(t)} \leq & C^{*}\left(\omega(m)\left\|v^{\sigma, k}\right\|_{Y(t)}\right. \\
& \left.+K\left(2 D_{m} \epsilon\left\|v^{\sigma}\right\|_{Y(t)}+\left(2 D_{m} C_{\epsilon}+E_{m}\right)\left\|v^{\sigma}\right\|_{X(t)}\right)+\left\|f^{k}\right\|_{X}\right) .
\end{aligned}
$$

Fix $m$ large enough such that $C^{*} \omega(m) \leq \frac{1}{2}$. Then (2.24) implies

$$
\left\|v^{\sigma, k}\right\|_{Y(t)} \leq \epsilon C_{00}\left\|v^{\sigma}\right\|_{Y(t)}+C_{01}(\epsilon)\left\|v^{\sigma}\right\|_{X(t)}+2 C^{*}\left\|f^{k}\right\|_{X}
$$

where $C_{00}$ and $C_{01}(\epsilon)$ also depend on $m, K, C^{*}$. Summing over $k$, we obtain $(2.26)$

$\left\|v^{\sigma}\right\|_{Y(t)} \leq \sum_{k=1}^{N(m)}\left\|v^{\sigma, k}\right\|_{Y(t)} \leq C_{02}\|f\|_{X}+\epsilon N(m) C_{00}\left\|v^{\sigma}\right\|_{Y(t)}+N(m) C_{01}(\epsilon)\left\|v^{\sigma}\right\|_{X(t)}$ 
with $C_{02}$ depending on the same quantities as $C_{00}$ and $C_{01}$. Choose $\epsilon$ so small that $\epsilon N(m) C_{00} \leq \frac{1}{2}$, then

$$
\left\|v^{\sigma}\right\|_{Y(t)} \leq 2 C_{02}\|f\|_{X}+C_{03}\left\|v^{\sigma}\right\|_{X(t)}
$$

with $C_{03}$ still depending only on $C^{*}, n,\|B\|_{\infty}$, and the modulus of continuity of $B$. Since $v^{\sigma}(\cdot, 0)=0$, we have for any $t$

$$
\left\|v^{\sigma}\right\|_{X(t)} \leq C(T, p) \int_{0}^{t}\left\|v^{\sigma}\right\|_{Y(s)} d s
$$

Inserting (2.28) in (2.27) we obtain

$$
\left\|v^{\sigma}\right\|_{Y(t)} \leq 2 C_{02}\|f\|_{X}+C_{03} C(T, p) \int_{0}^{t}\left\|v^{\sigma}\right\|_{Y(s)} d s
$$

from which

$$
\left\|v^{\sigma}\right\|_{Y(T)} \leq C_{04}\|f\|_{X}
$$

follows by an application of Gronwall's inequality, with $C_{04}$ not depending on $\sigma$. By the argument that was given earlier, the lemma follows.

Proof of Theorem 2.2. Let $C_{1}>0$ be such that estimate (2.2) holds with this constant for all solutions of

$$
w_{t}(x, t)-\int_{0}^{t} a(t-s)\left(B(x, \sigma): \nabla^{2} w(x, s)+f(x, s)\right) d s=0
$$

where $0 \leq \sigma \leq T$. Such a common constant exists by the previous lemma and the assumptions for $B$. Let $N \in \mathbf{N}$ be so large that for all $0 \leq s, t \leq T$ with $|s-t| \leq \frac{T}{N}$

$$
C_{1}\|B(\cdot, t)-B(\cdot, s)\|_{\infty} \leq \frac{1}{2} .
$$

For $k=1, \ldots, N$ and $x \in \bar{\Omega}$ define

$$
B^{k}(x, t)=\left\{\begin{array}{lc}
B(x, t) & \left(t \leq \frac{k T}{N}\right), \\
B\left(x, \frac{k T}{N}\right) & \left(t>\frac{k T}{N}\right) .
\end{array}\right.
$$

Now define $v^{1} \in Y$ to be the solution on $\Omega \times[0, T]$ of

$$
v_{t}^{1}(x, t)-\int_{0}^{t} a(t-s)\left(B^{1}(x, s): \nabla^{2} v^{1}(x, s)+f(x, s)\right) d s=0
$$

with initial data $v^{1}(\cdot, 0)=0$. Since $C_{1}\left\|B^{1}(\cdot, t)-B^{1}(\cdot, 0)\right\|_{\infty} \leq \frac{1}{2}$ by the choice of $N$, Theorem 2.1 implies that $v^{1}$ is well-defined. Next define for $k=2, \ldots, N$ the functions $v^{k} \in Y$ as solutions of the problems

$$
\begin{aligned}
& v_{t}^{k}(x, t)-\int_{\frac{k-1}{N} T}^{t} a(t-s)\left(B^{k}(x, s): \nabla^{2} v^{k}(x, s)+f^{k}(x, s)\right) d s=0 \\
&\left(x \in \Omega, \frac{k-1}{N} T<t \leq T\right)
\end{aligned}
$$


where

$$
f^{k}(x, s)=\left(B^{k}(x, s)-B^{k-1}(x, s)\right): \sum_{j=1}^{k-1} \nabla^{2} v^{j}(x, s),
$$

with inital data $v^{k}\left(\cdot, \frac{k-1}{N} T\right)=0$. By (2.31) and (2.32), the $B^{k}$ are small perturbations of $B^{k}\left(\cdot, \frac{k-1}{N} T\right)$, and Theorem 2.1 again implies that all $v^{k}$ are well-defined. Also, since $f^{k}(\cdot, t)$ vanishes for $t \leq \frac{k-1}{N} T$, we can continue each $v^{k}$ as zero on $\bar{\Omega} \times\left[0, \frac{k-1}{N} T\right]$ and change the lower limit of the integration to 0 , and (2.34) still holds. A trivial application of Theorem 2.1 also implies

$$
\left\|v^{1}\right\|_{Y} \leq 2 C_{1}\|f\|_{X}
$$

and

$$
\left\|v^{k}\right\|_{Y} \leq C_{k} \cdot \sum_{j=1}^{k-1}\left\|v^{j}\right\|_{Y}
$$

for all $k \geq 2$, with suitable constants. Set $v=\sum_{j=1}^{N} v^{j}$, then $v$ solves $(2.1 \mathrm{a}, \mathrm{b}, \mathrm{c})$, and (2.2) holds. The theorem is proved.

\section{Estimates For SPATial DERIVATIVEs}

In this section, we prove the following pointwise estimate for solutions of $(1.1 \mathrm{a}, \mathrm{b}, \mathrm{c})$. All assumptions and the notation of the previous two section are to hold.

Lemma 3.1. Let $u$ be a solution of $(1.1 a, b, c)$, and let the assumptions of Theorem 1.1 hold. Then there exist $\delta>0$, depending only on $\alpha$, and $C>0$, depending on the data, such that for any $(x, t),(y, s) \in I \times[0, T]$

$$
\left|u_{x}(x, t)-u_{x}(y, s)\right| \leq C\left(|t-s|^{\delta}+|x-y|^{\delta}\right) .
$$

Of course, an estimate like (3.1) holds for any solution whose existence is asserted in Theorem 1.1. The point of the lemma is that $\delta$ and $C$ depend only on the data. We begin by showing an a priori estimate for some integral norm of $\psi\left(u_{x}\right)$. This can be done without restricting $\alpha$. Here and below, the usual notation for spaces of Hölder continuous functions is employed. Thus let now $2 p^{-1}+q^{-1}<1$, and let $u$ be a solution of $(1.1 \mathrm{a}, \mathrm{b}, \mathrm{c})$ with $u_{x x t} \in L^{p}\left(0, T ; L^{q}(I)\right)$. Let $\delta_{0}>0$ be as in (1.15).

Lemma 3.2. If $0<\alpha<1$, then there is a constant $C=C\left(f, u_{0}, u_{1}, a, \varphi, \psi\right)$ such that for all $0 \leq t \leq T$

$$
\left\|u_{t}(\cdot, t)\right\|_{2}^{2}+\int_{t-\delta_{0}}^{t}(t-s)^{-1-\alpha}\left\|\psi\left(u_{x}(\cdot, t)\right)-\psi\left(u_{x}(\cdot, s)\right)\right\|_{2}^{2} d s \leq C .
$$

Here $\psi\left(u_{x}(\cdot, s)\right)=\psi\left(u_{0, x}\right)$ for $s<0$.

Proof. After an integration by parts, (1.1a) appears in the form

$$
u_{t t}(x, t)-\varphi\left(u_{x}(x, t)\right)_{x}+\int_{-\infty}^{t} a^{\prime}(t-s)\left(\psi\left(u_{x}(x, t)\right)-\psi\left(u_{x}(x, s)\right)\right)_{x} d s=f(x, t) .
$$


Multiply with $u_{t}(x, t)$, integrate over $I$, and integrate by parts. The result is the identity

$$
\frac{d}{d t}\left(\frac{1}{2}\left\|u_{t}(\cdot, t)\right\|_{2}^{2}+\Phi\left(u_{x}(\cdot, t)\right)\right)-\int_{-\infty}^{t} a^{\prime}(t-s) \frac{d}{d t} H(t, s) d s=\int_{I} f(\cdot, t) u_{t}(\cdot, t),
$$

where

$$
\begin{aligned}
\Phi(t) & =\int_{I} \int_{0}^{u_{x}(x, t)} \varphi(r) d r d x, \\
H(t, s) & =\int_{I} h\left(u_{x}(x, t), u_{x}(x, s)\right) d x, \\
h(v, w) & =\int_{w}^{v}(\psi(r)-\psi(w)) d r .
\end{aligned}
$$

Note that by Lemma A.3, $u_{x t}$ is continuous on $I \times[0, T]$ and thus

$$
H(t, s)=O\left(|t-s|^{2}\right), \quad \frac{d}{d t} H(t, s)=O(|t-s|),
$$

uniformly in $0 \leq s, t \leq T$, with of course no information about the constants. Also, $\Phi(t) \geq-L$ for all $t$ by (1.16), and $H(t, s) \geq 0$ for all $s, t$, since $\psi$ is assumed to be strictly increasing. Then (3.3) can be rewritten as

$$
\begin{aligned}
\frac{d}{d t}\left(\frac{1}{2}\left\|u_{t}(\cdot, t)\right\|_{2}^{2}\right. & \left.+\Phi(t)-\int_{-\infty}^{t} a^{\prime}(t-s) H(t, s) d s\right) \\
& =-\int_{-\infty}^{t} a^{\prime \prime}(t-s) H(t, s) d s+\int_{I} f(\cdot, t) u_{t}(\cdot, t) .
\end{aligned}
$$

Using (1.14), the right-hand side of this identity can be estimated from above by

$$
M \int_{-\infty}^{t}\left(-a^{\prime}(t-s)\right) H(t, s) d s+\int_{I}\|f(\cdot, t)\|_{2}\left\|u_{t}(\cdot, t)\right\|_{2} .
$$

Integrating now from 0 to $t$ and using Bihari's inequality, we obtain the estimate

$$
\frac{1}{2}\left\|u_{t}(\cdot, t)\right\|_{2}^{2}+\int_{-\infty}^{t}\left(-a^{\prime}(t-s)\right) H(t, s) d s \leq C
$$

for $0 \leq t \leq T$, where $C$ depends only on the data. The desired estimate now follows from assumption (1.15) and from the following simple property of $h$.

Lemma 3.3. Let $\psi: \mathbf{R} \rightarrow \mathbf{R}$ be increasing with

$$
|\psi(u)-\psi(v)| \leq L|u-v|
$$

for some $L>0$ for all $u, v \in \mathbf{R}$. Then for all $u, v \in \mathbf{R}$

$$
h(u, v)=\int_{v}^{u}(\psi(r)-\psi(v)) d r \geq(2 L)^{-1}|\psi(u)-\psi(v)|^{2} .
$$


Proof. If $\psi$ is continuously differentiable, then for $r \geq v$

$$
\psi(r)-\psi(v) \geq L^{-1} \psi^{\prime}(r)(\psi(r)-\psi(v))
$$

and the reverse inequality holds for $r \leq v$. Integrating from $v$ to $u$ gives the desired estimate in each case. If $\psi$ is merely continuous, the estimate follows by approximation.

We next derive a Hölder continuity estimate for the $L^{2}$ - valued function $t \rightarrow$ $\psi\left(u_{x}(\cdot, t)\right)$. The arguments are adapted from techniques in the theory of generalized Lipschitz (Hölder) spaces, see [2].

Lemma 3.4. There is a constant $C=C\left(f, u_{0}, u_{1}, a, \varphi, \psi\right)$ such that for all $s, t, \in$ $[0, T]$

$$
\left\|\psi\left(u_{x}(\cdot, s)\right)-\psi\left(u_{x}(\cdot, t)\right)\right\|_{2} \leq C|t-s|^{\alpha / 2} .
$$

Proof. It suffices to show the estimate for $|t-s| \leq \delta_{0}$. We write $w(t)=\psi\left(u_{x}(\cdot, t)\right) \in$ $L^{2}(I)$ for $t \leq T$, continued as $\psi\left(u_{0, x}\right)$ for $t<0$, and set

$$
w_{h}(t)=\frac{2}{h^{2}} \int_{t-h}^{t}(s-t+h) w(s) d s
$$

for $0<h<\delta_{0}$. From Lemma 3.2 and Hölder's inequality we obtain for $0 \leq t \leq T$

$$
\begin{aligned}
\int_{t-h}^{t} & \|w(t)-w(s)\|_{2} d s \\
& \leq\left(\int_{0}^{h} s^{1+\alpha} d s\right)^{1 / 2}\left(\int_{t-h}^{t}(t-s)^{-1-\alpha}\|w(t)-w(s)\|_{2}^{2} d s\right)^{1 / 2} \leq C h^{1+\alpha / 2} .
\end{aligned}
$$

Consequently

$$
\begin{aligned}
\left\|w(t)-w_{h}(t)\right\|_{2} & =\left\|\frac{2}{h^{2}} \int_{t-h}^{t}(s-t+h)(w(t)-w(s)) d s\right\|_{2} \\
& \leq \frac{2}{h} \int_{t-h}^{t}\|w(t)-w(s)\|_{2} d s \\
& \leq C h^{\alpha / 2}
\end{aligned}
$$

and

$$
\left\|w_{h}^{\prime}(t)\right\|_{2} \leq \frac{2}{h^{2}} \int_{t-h}^{t}\|w(t)-w(s)\|_{2} d s \leq C h^{\alpha / 2-1} .
$$

Thus for such $h$ and for $0 \leq s \leq t \leq s+\delta_{0} \leq T$

$$
\begin{aligned}
\|w(t)-w(s)\|_{2} & \leq\left\|w(t)-w_{h}(t)\right\|_{2}+\left\|w_{h}(t)-w_{h}(s)\right\|_{2}+\left\|w_{h}(s)-w(s)\right\|_{2} \\
& \leq C\left(h^{\alpha / 2}+|t-s| h^{\alpha / 2-1}\right) .
\end{aligned}
$$

Setting $h=|t-s|$ proves the lemma. 
With these preparations, the proof of Lemma 3.1 can now be completed. Let $\alpha$ be restricted to the range $2 / 3<\alpha<1$, and let

$$
b=\frac{\sin \alpha \pi}{\pi}\left(a_{1-\alpha}+d * a_{1-\alpha}\right)
$$

where $b \in L^{1}(0, T ; \mathbf{R})$ is the integral resolvent of the kernel $a=a_{\alpha}+c * a_{\alpha}$ as in Lemma A.2, i.e.

$$
\int_{0}^{t} a(t-s) b(s) d s=1
$$

for all $t \geq 0$. We define the auxiliary function

$$
v(x, t)=\int_{0}^{t}\left(\psi\left(u_{x}(x, s)\right)+\int_{0}^{s} b(s-\tau) \varphi\left(u_{x}(x, \tau)\right) d \tau\right) d s .
$$

Assumption (1.16) implies that $\varphi$ is a Lipschitz continuous function of $\psi$. Since $c \in L^{1}$, Lemma 3.4 implies that $v \in C^{1, \alpha / 2}\left([0, T], L^{2}(I)\right)$ with its norm in this space estimated by the data. On the other hand, after integrating (1.1a) with respect to $t$ and forming the convolution of the result with $b$, one sees that $v$ also satisfies the equation

$$
v_{x}(x, t)=\int_{0}^{t} b(t-s)\left(u_{t}(x, s)-u_{1}(x)\right) d s+\int_{0}^{t} f_{1}(x, s) d s
$$

with $f_{1} \in L^{\infty}\left(0, T ; L^{2}(I, \mathbf{R})\right)$ depending only on the data. It is known that convolution with $b$ maps $L^{\infty}(0, T ; X)$ continuously into $C^{\alpha}([0, T], X)$, for any Banach space $X([12])$. By the estimate for $u_{t}$ in Lemma 3.2, the right-hand side in this identity thus can be estimated a priori in $C^{\alpha}\left([0, T], L^{2}(I)\right)$. Integrating for fixed $t$ with respect to $x$ from $\xi \in[0,1]$ to $y \in[0,1]$ and then with respect to $\xi$ over $[0,1]$ implies that $v \in C^{\alpha}\left([0, T], H^{1}(I)\right)$, and its norm in this space can be estimated from the data.

Since Hölder properties can be characterized by means of iterated difference quotients $([2])$, these continuity properties of $v$ are equivalent to

$$
\|v(\cdot, t-h)+v(\cdot, t+h)-2 v(\cdot, t)\|_{L^{2}} \leq C h^{1+\alpha / 2}
$$

and

$$
\|v(\cdot, t-h)+v(\cdot, t+h)-2 v(\cdot, t)\|_{H^{1}} \leq C h^{\alpha}
$$

for all $h \leq t \leq T-h$ and all small $h$. Next recall the interpolation inequality

$$
\|w\|_{H^{\sigma}} \leq C\|w\|_{H^{1}}^{\sigma}\|w\|_{L^{2}}^{1-\sigma}
$$

for all $w \in H^{1}$ ([1], [3]). Choose $\sigma \in\left(\frac{1}{2}, \frac{\alpha}{2-\alpha}\right)$. This is possible, since $\alpha>2 / 3$. Then also

$$
\|v(\cdot, t-h)+v(\cdot, t+h)-2 v(\cdot, t)\|_{H^{\sigma}} \leq C h^{\delta+1}
$$

where

$$
\delta=\sigma \alpha+(1-\sigma)\left(1+\frac{\alpha}{2}\right)-1>0
$$


Thus $v$ can also be estimated a priori in the space $C^{1, \delta}\left([0, T], H^{\sigma}(I)\right)$, in terms of the data. Since $\left.H^{\sigma}(I)\right) \subset C^{\sigma-1 / 2}(I)$ with continuous imbedding ([1]), we obtain that $v_{t} \in C^{\delta}\left([0, T], C^{\sigma-1 / 2}(I)\right)$ with an estimate in this space. Recall next that

$$
v_{t}(x, t)=\psi\left(u_{x}(x, t)\right)+\int_{0}^{t} b(t-s) F\left(\psi\left(u_{x}(x, s)\right)\right) d s
$$

with some Lipschitz continuous function $F$. Standard results on Volterra integral equations ([14]) imply that also $\psi\left(u_{x}\right) \in C^{\delta}\left([0, T], C^{\sigma-1 / 2}(I)\right)$ together with an estimate. Finally, since $\psi: \mathbf{R} \rightarrow \mathbf{R}$ is onto and $\psi^{-1}$ is Hölder continuous, it follows that also $u_{x}$ is in such a Hölder space. This proves Lemma 3.1.

\section{Existence PROOF}

We now give the proof of the existence theorem 1.1. With $p, q$ satisfying $2 p^{-1}+$ $q^{-1}<1$ and the spaces $X$ and $\tilde{Y}$ as in section 1 , we define the space $Z$ by

$$
Z=\left\{u: T \times[0, T] \rightarrow \mathbf{R} \mid u, u_{t} \in \tilde{Y}\right\}
$$

with its natural norm. Then Lemma A.3 implies that $z_{x t}$ is still Hölder continuous for any $z \in Z$, and the imbedding from $Z$ into the space

$$
C^{1,1}=\left\{u: T \times[0, T] \rightarrow \mathbf{R} \mid u_{x t} \quad \text { is continuous }\right\}
$$

with its natural norm is completely continuous. Let $a \in K(\alpha)$ with $\alpha>\frac{2}{3}$, and let $b$ be the kernel for which $a * b(t)=1$, as determined in Lemma A.2.

Let $u_{0}, u_{1} \in Y_{0, q}(\Omega)$ and $f$ as in (1.17) be given. For $u \in Z$ and $0 \leq \sigma \leq 1$, we define

$$
w^{\sigma}(x, t)=\left\{\begin{array}{l}
u_{x}(x, t) \quad(0 \leq t \leq \sigma T), \\
u_{x}(x, \sigma T)+(t-\sigma T) u_{x t}(x, \sigma T) \quad(\sigma T<t \leq T)
\end{array}\right.
$$

with time derivative $w_{t}^{\sigma}$. The functions $w^{\sigma}$ and $w_{t}^{\sigma}$ are continuous on $I \times[0, T]$. Then let $v \in \tilde{Y}$ be the unique solution of the equation

$v_{t}(x, t)-\int_{0}^{t} a(t-s)\left(\psi^{\prime}\left(w^{\sigma}(x, s)\right) v_{x x}(x, s)+\sum_{i=1}^{3} N_{i}\left[w^{\sigma}\right](v)(x, s)+f_{2}(x, s)\right) d s=0$

with inital data $v(x, 0)=u_{1}(x)$. Here

$$
\begin{aligned}
& N_{1}\left[w^{\sigma}\right](v)(x, s)=\psi^{\prime \prime}\left(w^{\sigma}(x, s)\right) w_{t}^{\sigma}(x, s)\left(u_{0, x x}(x)+\int_{0}^{s} v_{x x}(x, \tau) d \tau\right), \\
& N_{2}\left[w^{\sigma}\right](v)(x, s)=\int_{0}^{s} b(s-\tau) \varphi^{\prime}\left(w^{\sigma}(x, \tau)\right) v_{x x}(x, \tau) d \tau, \\
& N_{3}\left[w^{\sigma}\right](v)(x, s) \\
& \quad=\int_{0}^{s} b(s-\tau) \varphi^{\prime \prime}\left(w^{\sigma}(x, \tau)\right) w_{t}^{\sigma}(x, \tau)\left(u_{0, x x}(x)+\int_{0}^{\tau} v_{x x}(x, \rho) d \rho\right) d \tau,
\end{aligned}
$$


and

$$
f_{2}(x, t)=\left\{\begin{array}{l}
b(s) \varphi\left(u_{0, x}(x)\right)_{x}+f_{0}(x, t) \quad \text { if } \quad p(1-\alpha)<1, \\
f_{0}(x, t) \quad \text { if } p(1-\alpha)>1 .
\end{array}\right.
$$

We then define $V=\mathbf{S}(\sigma, u)$ by

$$
V(x, t)=u_{0}(x)+\int_{0}^{t} v(x, \tau) d \tau .
$$

The operator $\mathbf{S}$ is well-defined for all $\sigma$ and maps $Z$ into itself. Indeed, $\psi^{\prime}\left(w^{\sigma}(\cdot)\right)$ is positive and continuous, the function $f_{2}$ belongs to $X$ for all possible $p$, and the integral operators $N_{i}$ all qualify as lower order terms in the sense of Theorem 2.3.

The next thing to notice is that $u \in Z$ is a solution of (1.1a) on $I \times[0, \sigma T]$ if and only if $u=\mathbf{S}(\sigma, u)$ on this set. In particular, a solution of the full problem (1.1a,b,c) is a fixed point of the operator $\mathbf{S}(1, \cdot)$. Finally, an inspection of all terms in the definition of $\mathbf{S}$ shows that the mapping is completely continuous in $u \in Z$, due to the compactness of the imbedding from $Z$ to $C^{1,1}$ (Lemma A.3) and the results on continuous dependence on coefficients in section 2, in particularly Theorem 2.3.

We now claim that there is a constant $M$, depending only on the data, such that for any fixed point $u$ of $S(\sigma, \cdot)$ the following estimate holds:

$$
\|u\|_{Z} \leq M \text {. }
$$

For $\sigma=0$, the estimate is clear; thus let $\sigma>0$. Then $u$ satisfies (1.1) on $I \times[0, \sigma T]$. It follows from Lemma 3.1 that $u_{x}$ is in some compact subset of $C(I \times[0, \sigma T], \mathbf{R})$ that does not depend on $\sigma$. This means that for all such fixed points, the $u_{x}$ are uniformly bounded and have some common modulus of continuity. Since $w$, defined by $w(x, t)=u(x, t)-t u_{1}(x)$, satisfies the equation

$$
\begin{array}{r}
w_{t}(x, t)-\int_{0}^{t} a(t-s)\left(\psi^{\prime}\left(u_{x}(x, s)\right) w_{x x}(x, s)+b * \varphi^{\prime}\left(u_{x}(x, s)\right) w_{x x}(x, s)\right. \\
\left.+f_{3}(x, s)\right) d s=0
\end{array}
$$

on $I \times[0, \sigma T]$ with

$$
\begin{aligned}
f_{3}(x, t)= & \int_{0}^{t} f_{2}(x, s) d s+t \psi^{\prime}\left(u_{x}(x, t)\right) u_{1, x x}(x) \\
& +\int_{0}^{t} b(t-s) s \varphi\left(u_{x}(x, s)\right) u_{1, x x}(x) d s-\psi\left(u_{0, x}(x)\right)_{x},
\end{aligned}
$$

we can again apply the results of section 2 and infer that $w$ and therefore also $u$, restricted to $I \times[0, \sigma T]$, are in some a priori bounded subset of $\tilde{Y}(\sigma T)$ with the bound independent of $\sigma$. Returning to (4.2) which holds for $v=u_{t}$ on $I \times[0, \sigma T]$, we rewrite the terms with $N_{1}$ and $N_{3}$ on this set in the form

$$
\begin{aligned}
& N_{1}\left[w^{\sigma}\right](v)(x, s)=\psi^{\prime \prime}\left(u_{x}(x, s)\right) v_{x}(x, s) u_{x x}(x, s), \\
& N_{3}\left[w^{\sigma}\right](v)(x, s)=\int_{0}^{s} b(s-\tau) \varphi^{\prime \prime}\left(u_{x}(x, \tau)\right) v_{x}(x, \tau) u_{x x}(x, \tau) d \tau
\end{aligned}
$$


and replace $w^{\sigma}$ with $u_{x}$ in $N_{2}$. Corollary 2.5 now implies that the function $v=u_{t}$ is also bounded in $\tilde{Y}(\sigma T)$, with a bound that is independent of $\sigma$. That is, $u$ is bounded in $Z(\sigma T)$. Finally, to deduce a bound on $I \times[0, T]$, we return to $(4.2)$ to see that $u$ satisfies a linear equation on $I \times[\sigma T, T]$ with uniformly bounded equicontinuous coefficients. A bound for $u$ in $Z(T)$ follows immediately.

We now use a standard topological degree argument (see [8]). Let

$$
\operatorname{deg}\left(\mathbf{S}(\sigma, \cdot)-I d, B_{M+1}(0), 0\right)
$$

denote the usual mapping degree of the mapping $\mathbf{S}(\sigma, \cdot)-I d: Z \rightarrow Z$, with $M$ as in (4.5). This is well-defined, since $\mathbf{S}(\sigma, \cdot)$ is completely continuous. Also, $\mathbf{S}$ clearly is jointly continuous in both arguments. Since $\mathbf{S}(0, \cdot)$ has exactly one fixed point and since the fixed point set of $S(\sigma, \cdot)$ never meets the set $\left\{z \in Z \mid\|z\|_{Z}=M+1\right\}$ for $0 \leq \sigma \leq 1$, we obtain

$$
\operatorname{deg}\left(\mathbf{S}(0, \cdot)-I d, B_{M+1}(0), 0\right)=\operatorname{deg}\left(\mathbf{S}(1, \cdot)-I d, B_{M+1}(0), 0\right)=1,
$$

i.e. $\mathbf{S}(1, \cdot)$ has at least one fixed point $u \in Z$. This fixed point is the desired solution.

\section{UNIQUENESS}

Uniqueness of solutions of quasilinear equations can be proved for a larger class of solutions than the one in which we prove global existence and for the entire range of singular kernels $a \in K(\alpha), 0<\alpha<1$. This is essentially the same situation in which one could also prove local existence of solutions, using Banach's Fixed Point Theorem.

Theorem 5.1. Let $a \in K(\alpha)$ with $0<\alpha<1$. Let $B: \Omega \times[0, T] \times \mathbf{R} \times \mathbf{R}^{n} \rightarrow$ $\mathbf{R}_{\text {sym }}^{n \times n}$ be continuous with positive definite values everywhere, and assume that for any $M>0$ there exists $L_{M}>0$ such that for all $(x, t) \in \Omega \times[0, T]$ and all $u_{1}, u_{2} \in \mathbf{R}, p_{1}, p_{2} \in \mathbf{R}^{n}$ with $\left|u_{i}\right|,\left\|p_{i}\right\| \leq M$ the following estimate holds:

$$
\left\|B\left(x, t, u_{1}, p_{1}\right)-B\left(x, t, u_{2}, p_{2}\right)\right\| \leq L_{M}\left(\left|u_{1}-u_{2}\right|+\left\|p_{1}-p_{2}\right\|\right) .
$$

Let $\frac{2}{p}+\frac{n}{q}<1$, and let $u_{0} \in Y_{0, q}(\Omega), f \in X_{p, q}(T, \Omega)$. Then the problem

$$
u_{t}(x, t)-\int_{0}^{t} a(t-s)\left(B(x, s, u(x, s), \nabla u(x, s)): \nabla^{2} u(x, s)+f(x, s)\right) d s=0
$$

with initial data $u(x, 0)=u_{0}$ in $\Omega$ has at most one solution $u \in \tilde{Y}$.

Proof. Suppose that $u, v \in \tilde{Y}$ are two solutions of the same equation (5.1). Set $w=u-v$, then $w \in Y$ solves

$$
w_{t}(x, t)-\int_{0}^{t} a(t-s)\left(B_{0}(x, s): \nabla^{2} w(x, s)+h(x, s)\right) d s
$$

with

$$
\begin{gathered}
B_{0}(x, s)=B(x, s, u(x, s), \nabla u(x, s)), \\
h(x, s)=(B(x, s, u(x, s), \nabla u(x, s))-B(x, s, v(x, s), \nabla v(x, s))): \nabla^{2} v(x, s) .
\end{gathered}
$$


By Lemma A.3, $\nabla u$ and $\nabla v$ are continuous and therefore bounded on $\bar{\Omega} \times[0, T]$. The function $B_{0}$ is therefore continuous on $\bar{\Omega} \times[0, T]$ and has positive definite values there, and there exists a constant $L$ such that

$$
\|h\|_{X(t)} \leq L\left(\|w\|_{L^{\infty}(t)}+\|\nabla w\|_{L^{\infty}(t)}\right)\|v\|_{Y} .
$$

As in the proof of Corollary 2.5, we have estimates

$$
\|w\|_{L^{\infty}(t)}+\|\nabla w\|_{L^{\infty}(t)} \leq \epsilon\|w\|_{Y(t)}+C_{\epsilon} \int_{0}^{t}\|w\|_{Y(s)} d s
$$

for any $\epsilon>0$, with some constant $C_{\epsilon}$. Applying Theorem 2.2, we obtain for any $0 \leq t \leq T$

$$
\|w\|_{Y(t)} \leq C_{0}\|h\|_{X(t)} \leq C_{1}\left(\epsilon\|w\|_{Y(t)}+C_{\epsilon} \int_{0}^{t}\|w\|_{Y(s)} d s\right)
$$

for some $C_{1}>0$. Choosing $\epsilon<\frac{1}{2 C_{1}}$ results in

$$
\|w\|_{Y(t)} \leq 2 C_{1} C_{\epsilon} \int_{0}^{t}\|w\|_{Y(s)} d s
$$

and Gronwall's Lemma then shows that $\|w\|_{Y}=0$, i.e. $u=v$ on $\Omega \times[0, T]$.

The proof obviously generalizes to problems with several integral terms such as the one for which existence of a solution in $\tilde{Y}$ was proved in the previous section. Only changes in the notation are required. Note that uniqueness is proved for a range of problems that strictly includes the class for which global existence is asserted, namely essentially for solutions whose spatial gradients are still continuous. The solutions that are constructed in this paper have time derivatives with continuous spatial gradients.

\section{APPENDIX}

Let $0<\alpha<1$. We recall the notation $a_{\alpha}(t)=t^{-\alpha}$ and

$$
K(\alpha)=\left\{a:(0, T] \rightarrow \mathbf{R} \mid a(t)=a_{\alpha}(t)+c * a_{\alpha}(t), c \in L^{1}(0, T ; \mathbf{R})\right\} .
$$

Lemma A.1. If $a(t)=d(t) \cdot a_{\alpha}(t)$ with $d \in W^{1,1}([0, T], \mathbf{R}), d(0)=1$, then $a \in$ $K(\alpha)$.

Proof. Assume first that $d$ is continuously differentiable. Then ([12])

$$
\int_{0}^{t} a_{\alpha}(t-s) a_{1-\alpha}(s) d s=\Gamma(\alpha) \Gamma(1-\alpha)=\frac{\pi}{\sin \pi \alpha} .
$$

Set $c_{\alpha}=\frac{\sin \pi \alpha}{\pi}$, and define

$$
c(t)=c_{\alpha} \int_{0}^{1}(1-\tau)^{\alpha-1} \tau^{1-\alpha} d^{\prime}(t \tau) d \tau .
$$


Then $c$ is defined everywhere and continuous, and

$$
\begin{aligned}
\|c\|_{L^{1}} & \leq c_{\alpha} \int_{0}^{T} \int_{0}^{1}(1-\tau)^{\alpha-1} \tau^{1-\alpha}\left|d^{\prime}(t \tau)\right| d \tau d t \\
& =c_{\alpha} \int_{0}^{1}(1-\tau)^{\alpha-1} \tau^{-\alpha} \int_{0}^{T \tau}\left|d^{\prime}(t)\right| d t d \tau \\
& \leq\left\|d^{\prime}\right\|_{L^{1}} .
\end{aligned}
$$

Also, for such a $C^{1}$ - function $d$,

$$
\begin{aligned}
c(t) & =\frac{d}{d t}\left(c_{\alpha} \int_{0}^{1}(1-\tau)^{\alpha-1} \tau^{-\alpha} d(t \tau) d \tau\right) \\
& =\frac{d}{d t}\left(c_{\alpha} \int_{0}^{t}(t-\tau)^{\alpha-1} \tau^{-\alpha} d(\tau) d \tau\right) \\
& =\frac{d}{d t}\left(c_{\alpha} \int_{0}^{t} a_{1-\alpha}(t-\tau) a_{\alpha}(\tau) d(\tau) d \tau\right)
\end{aligned}
$$

and thus

$$
\begin{aligned}
\int_{0}^{t} c(s) d s & =c_{\alpha} \int_{0}^{t} a_{1-\alpha}(t-s) \int_{0}^{s} a_{\alpha}(s-\tau) c(\tau) d \tau d s \\
& =c_{\alpha} \int_{0}^{t} a_{1-\alpha}(t-\tau) a_{\alpha}(\tau)(d(\tau)-1) d \tau .
\end{aligned}
$$

Standard results about Abel integral equations ([12]) then imply that

$$
\int_{0}^{t} a_{\alpha}(t-s) c(s) d s=a_{\alpha}(t)(d(t)-1)
$$

for all $t$. A general function $d \in W^{1,1}$ now can be approximated by $C^{1}$ - functions, and a passage to the limit in the above arguments shows that $c$ must be integrable also in this case and that the required identity holds.

Lemma A.2. Let $a \in K(\alpha)$. Then there exists a kernel $b$ such that

$$
\int_{0}^{t} a(t-s) b(s) d s=1
$$

for all $t$. If $a=a_{\alpha}+c * a_{\alpha}$ with $c \in L^{1}(0, T ; \mathbf{R})$, then $b=c_{\alpha}\left(a_{1-\alpha}+a_{1-\alpha} * d\right)$, where $d \in L^{1}(0, T ; \mathbf{R})$ such that $c+d+c * d=0$ and $c_{\alpha}=\frac{\sin \pi \alpha}{\pi}$ as before.

Proof. Direct calculation.

Lemma A.3. Let $\frac{2}{p}+\frac{n}{q}<1$, and let $\tilde{Y}_{p, q}(T, \Omega)$ be defined as in section 1 . Then

$$
\tilde{Y} \subset C^{1,0}=\left\{u: \Omega \times[0, T] \rightarrow \mathbf{R} \mid \nabla u \in C\left(\Omega \times[0, T], \mathbf{R}^{n}\right)\right\}
$$

with completely continuous imbedding.

Proof. Let $0<\epsilon<\frac{1}{2}\left(1-\frac{2}{p}-\frac{n}{q}\right)$. We show that $\tilde{Y}$ is contained in

$$
\left\{u \mid \nabla u \in C^{\epsilon}\left([0, T], L^{\infty}(\Omega)\right) \cap L^{\infty}\left(0, T ; C^{\epsilon}(\Omega)\right)\right\}
$$


with bounded imbedding. The imbedding of this space in $C^{1,0}$ is known to be completely continuous. Thus let $u \in Y$; then $u$ is also an element of the real interpolation spaces between $L^{p}\left(0, T ; W^{2, q} \cap W_{0}^{1, q}\right)$ and $W^{1, p}\left([0, T], L^{q}\right)([1],[33])$. Consequently,

$$
\nabla u \in W^{s_{1}, p}\left([0, T], W^{2 s_{2}-1, q}\right)
$$

for all $s_{1}+s_{2}<1, s_{2}>\frac{1}{2}$. Now pick $\delta \in\left(\epsilon, \frac{1}{2}-\frac{1}{p}-\frac{n}{2 q}\right)$, set $s_{1}=\delta+\frac{1}{p}$, and choose $s_{2} \in\left(\frac{1}{2}+\frac{n}{2 q}, 1-s_{1}\right)$. By well-known imbedding theorems $([1])$, we obtain that

$$
\nabla u \in C^{\epsilon}\left([0, T], W^{1-2 s_{2}, q}\right) \subset C^{\epsilon}\left([0, T], L^{\infty}\right)
$$

Similarly, we set $s_{2}=\frac{1}{2}+\frac{n}{2 q}+\frac{\delta}{2}$ and choose $s_{1} \in\left(\frac{1}{p}, 1-s_{2}\right)$. Then $W^{2 s_{2}-1, q}(\Omega) \subset$ $C^{\epsilon}(\Omega)$ and thus

$$
\nabla u \in W^{s_{1}, p}\left([0, T], C^{\epsilon}(\Omega)\right) \subset L^{\infty}\left(0, T ; C^{\epsilon}(\Omega)\right)
$$

This completes the proof.

The author would like to thank the referee for some useful suggestions.

\section{REFERENCES}

1. R. S. Adams, Sobolev Spaces., Academic Press, New York, 1975. MR 56:9247

2. H. Behrens \& P.L. Butzer, Semigroups of Operators and Approximation, Springer, Berlin, Heidelberg, New York, 1967. MR 37:5588

3. J. Bergh \& L. Löfström, Interpolation Spaces, Springer, Berlin, Heidelberg, New York, 1976. MR 58:2349

4. C.M. Dafermos \& J.A. Nohel, A nonlinear hyperbolic Volterra equation in viscoelasticity, Amer. J. Math. Supplement (1981), 87-116. MR 83f:45003

5. C.M. Dafermos, Development of singularities in the motion of materials with fading memory, Arch Rat. Mech. Anal. 91 (1985), 193-205. MR 87a:73033

6. __ Estimates for conservation laws with little viscosity, SIAM J. Appl. Math. 18 (1987), 409-421. MR 88h:35071

7. G. Da Prato, M. Ianelli \& E. Sinestrari, Regularity of solutions of a class of linear integrodifferential equations in Banach spaces, J. Integral Eq. 8 (1985), 27-40. MR 86g:45019

8. K. Deimling, Nichtlinear Gleichungen und Abbildungsgrade, Springer, Berlin, Heidelberg, New York, 1974. MR 58:17985

9. H. Engler, Weak solutions of a class of quasilinear hyperbolic integro-differential equations describing viscoelastic materials, Arch. Rat. Mech. Anal. 113 (1990), 1-38. MR 91k:35052

10. __ Strong solutions of quasilinear integrodifferential equations with singular kernels in several space dimensions, Electronic J. Differential Eq., vol. 1995, no. 2, pp. 1-16.

11. A. Friedman, Partial Differential Equations, Holt, Rinehart, Winston, New York, 1969. MR 56:3433

12. R. Gorenflo \& S. Vessella, Abel Integral Equations, Lecture Notes in Mathematics 1461, Springer, Berlin, Heidelberg, New York, Tokyo, 1991. MR 92e:45003

13. G. Gripenberg, Nonexistence of smooth solutions for shearing flows in a nonlinear viscoelastic fluid, SIAM J. Math. Anal. 13 (1982), 954-961. MR 83m:76007

14. G. Gripenberg, S.-O. Londen \& O. Staffans, Volterra Integral and Functional Equations, Cambridge University Press, Cambridge, 1990. MR 91c:45003

15. G. Gripenberg, Global existence of solutions of Volterra integrodifferential equations of parabolic type, J. Differential Eq. 102 (1993), 382-390. MR 94e:45007

16. __ On the uniqueness and nonuniqueness of weak solutions of hyperbolic-parabolic Volterra equations, Differential Integral Eq. 7 (1994), 509-522. MR 94k:45002

17. Nonlinear Volterra equations of parabolic type due to singular kernels, J. Differential Eq. 112 (1994), 154-169. MR 95c:45004 
18. _ Weak solutions of hyperbolic-parabolic Volterra equations, Trans. Amer. Math. Soc. 343 (1994), 675-694. MR 94i:45015

19. H. Hattori, Breakdown of smooth solutions in dissipative nonlinear hyperbolic equations, Quart. J. Appl. Math. 40 (1982), 113-127. MR 83j:35109

20. J. Heikonen, On the Existence of a Global Mild Solution for a Nonlinear Parabolic Integrodifferential Equation, Licentiate Thesis, Helsinki Univ. of Technology, Espoo, Finland, 1993.

21. W. Hrusa \& M. Renardy, On a class of quasilinear partial integrodifferential equations with singular kernels, J. Differential Eq. 64 (1986), 195-220. MR 88c:45010

22. __ A model equation for viscoelasticity with a strongly singular kernel, SIAM J. Math. Anal. 19 (1988), 257-269. MR 89d:35159

23. S. Klainerman \& A. Majda, Formation of singularities for wave equations including the nonlinear string equation, Comm. Pure Appl. Math. 33 (1980), 241-263. MR 81f:35080

24. J.-L. Lions, Quelques Méthodes de Resolution des Problèmes Non-Linéaires, Dunod, Paris, 1969. MR 41:4326

25. S.-O. Londen, An existence result on a Volterra equation in a Banach space, Trans. Amer. Math. Soc. 235 (1978), 285-304. MR 57:13432

26. __ Some existence results for a nonlinear hyperbolic integrodifferential equation with singular kernel, J. Integral Eq. Appl. 3 (1991), 3-30. MR 92a:45035

27. R. C. MacCamy, Existence, uniqueness and stability of solutions of the equation $u_{t t}=$ $\left(\sigma\left(u_{x}\right)+\lambda\left(u_{x}\right) u_{x t}\right)_{x}$, Indiana Univ. Math. J. 20 (1970), 231-238. MR 42:699

28. J. Prüss \& H. Sohr, On operators with bounded imaginary powers in Banach spaces, Math. Z. 203 (1990), 429-452. MR 91b:47030

29. J. Prüss, Quasilinear parabolic equations in spaces of integrable functions, Semigroup Theory and Evolution Equations (B. de Pagter, Ph. Clément, E. Mitidieri, eds.), Lecture Notes in Pure Appl. Math. 135, Marcel Dekker, New York, 1991, pp. 401-420. MR 92m:47001

30. _ Evolutionary Integral Equations and Applications, Birkhäuser, Basel, Boston, Berlin, 1993. MR 94h:45010

31. M. Renardy, W.E. Hrusa \& J.A. Nohel, Mathematical Problems in Viscoelasticity, Longman Group, Boston, London, Melbourne, 1987. MR 89b:35134

32. M. Renardy, Coercive estimates and existence of solutions for a model of one-dimensional viscoelasticity with a non-integrable memory function, J. Integral Eq. Appl. 1 (1988), 7-16. MR 89k:45009

33. E. M. Stein, Singular Integrals and Differentiability Properties of Functions, Princeton University Press, Princeton, 1970. MR 44:7280

Department of Mathematics, Georgetown University, Washington, D.C. 20057

E-mail address: engler@guvax.acc.georgetown.edu 